\title{
Improvement of the whole lip contour by additional filler injection to the lateral vermilion zone: a report of three cases
}

\author{
Dae-jeong Park, $\mathrm{MD}^{1} \mathbb{1}$, Kyoungjin Kang, MD, PhD² \\ ${ }^{1}$ Toxnfill Clinic, Seoul, Rep. of Korea, ${ }^{2}$ Seoul Cosmetic Surgery Clinic, Busan, Rep. of Korea
}

\begin{abstract}
Hyaluronic acid (HA) fillers have been widely used to change the lip contour. Most techniques were focused on volume augmentation and wrinkle reduction. In young women, fillers are used for lip volume enhancement, while in older women, fillers are used for both lip volume and vermilion border enhancement. Until now, the most reliable method to improve the lateral contour of the upper lip has not been reported. This study aimed to improve the ptotic contour of the lateral lip by filler injection. HA filler was injected into the vermilion medial zone in the lower lip (approximately $0.2 \mathrm{ml}$ on each side) through the vermilion border and central vermilion through the dry and wet junction in the upper lip $(0.05-0.1 \mathrm{ml})$ for volume augmentation. Then, the filler was injected into the lateral vermilion zone between the flat and rolling-in parts in the upper lip through the upper vermilion border to improve ptotic appearance $(0.1 \mathrm{ml}$ on each side). Three patients underwent the procedure using the abovementioned method. The whole contour of the upper vermilion border was significantly changed from flat to round and curved. Even though the position of the mouth corner was not changed, it appeared lifted, which was caused by the illusion of eversion and anterior projection of the medial vermilion and changed contour of the lateral vermilion in the upper lip. The three patients were satisfied with their dynamic and vibrant-looking lips. Additional filler injection to the lateral vermilion zone to improve the whole contour of the lips was considered a relatively easy technique for three young patients.

Level of Evidence: IV
\end{abstract}

Keywords: hyaluronic acid filler; lip contour; mouth corner; ptotic

\section{Introduction}

Generally, the traditional lip augmentation technique was primarily focused on volume increase and wrinkle removal. Therefore, most fillers are injected into the central and medial vermilion zone and upper and lower vermilion borders, including the philtrum.

The satisfaction rate with the traditional technique was relatively high. However, some patients have frequently complained of unnatural contour with a ptotic appearance of the lateral vermilion zone and mouth corner after traditional lip augmentation procedure, in which the lip volume only increased in the central and medial vermilion zone (Fig. 1). Additionally, the mouth corners are also more inclined downward under the influence of the injected filler volume and muscles in the lower face when making an angry or sad expression [1]. It was believed that the ptotic contour with the rolling-in shape of the lateral vermilion border of the upper lip could not be techni-

Received May 29, 2019; Revised November 20, 2019; Accepted November 21, 2019

Corresponding author: Dae-jeong Park

E-mail: gnblsclinic@hanmail.net

This is an Open Access article distributed under the terms of the Creative Commons Attribution Non-Commercial License (http://creativecommons.org/licenses/by-nc/4.0), which permits unrestricted non-commercial use, distribution, and reproduction in any medium, provided the original work is properly cited.

Copyright $@ 2019$ Korean Society of Korean Cosmetic Surgery and Medicine (KSKCS \& KCCS). 

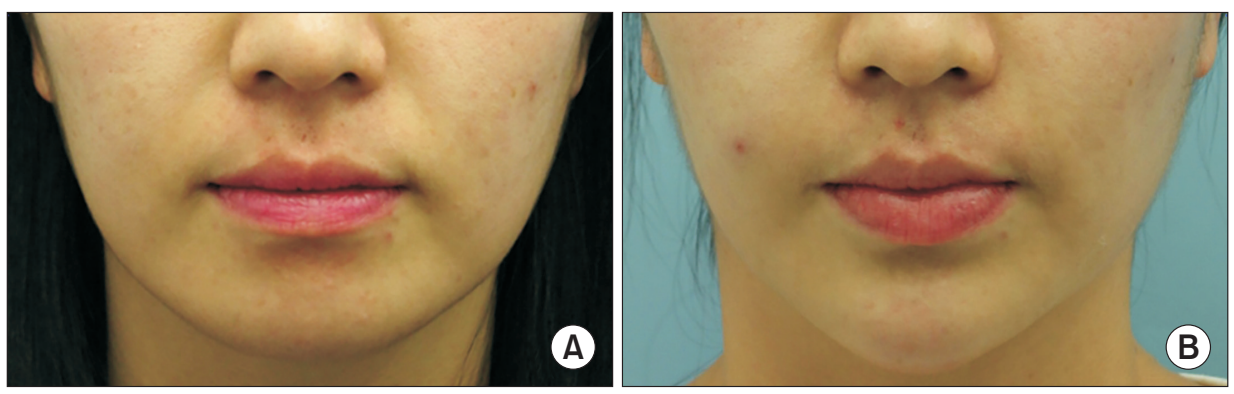

Fig. 1. Change in contour after traditional filler injection for lip augmentation in a young woman. Before (A) and after the procedure (B).

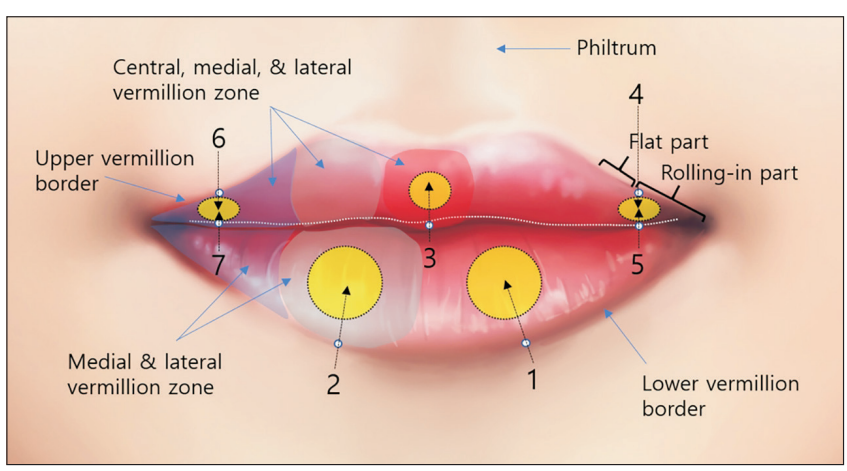

Fig. 2. Lip surface anatomy, markings, areas, and directions and particular order of injection. The numbers of 1, 2, 3, 4, 5, 6, and 7 indicate the injection sequence. Yellow-colored circles indicate volume injection areas. Black arrows indicate the directions of filler injection.

cally corrected by the traditional lip filler technique.

We attempted to inject the filler into not only the centromedial vermilion zone for volume restoration but also lateral vermilion zone to improve the ptotic contour of the lateral lip and mouth corner.

\section{Case report}

Lip filler injection was performed in three patients in the sitting position. Lidocaine cream (9.6\%) (Daehan New Pharm Co., Ltd., Hwasung, Korea) was applied on the surface of lips for 30 minutes. Povidone was applied to the surrounding area and the inside of the lips for sterilization purpose before the filler procedure. The filler used in the procedure was a type of hyaluronic acid (HA) filler, Juvéderm ${ }^{\circledR}$ VOLIFT $^{\mathrm{TM}}$ (Allergan, Courbevoie, France), and a 30-G sharp needle (4.0 mm in length) was used. The depth of all Injections was $4.0 \mathrm{~mm}$ (full needle size).

The lip surface anatomy, markings, areas, and directions and particular order of injection are shown in Fig. 2. Preoperative marking was performed on both sides of the upper vermilion border at approximately 0.5 to $0.8 \mathrm{~cm}$ from the mouth corner, in the middle of the wet-dry junction of the upper lip, and on both sides of the lower vermilion border approximately $1.0 \mathrm{~cm}$ lateral from the center of the lower lip.

While standing in front of the patient, we injected approximately $0.2 \mathrm{ml}$ of filler in the form of a bolus into the vermilion medial zone in the lower lip through its vermilion border and the same volume at the opposite side. Approximately 0.05 to 0.1 $\mathrm{ml}$ was injected in Cupid's bow for augmentation of the central vermilion border in the upper lip through the dry-wet junction [2].

While standing behind the patient, we injected approximately $0.1 \mathrm{ml}$ of filler into the junction between the flat and rolling-in parts of the lateral vermilion border parallel to the upper lip surface in the submucosal layer. At this time, the filler was injected with the downward bevel direction of the needle after performing the syringe regurgitation test. The thumb or index finger of the opposite hand was used to compress the upper part of the lip to prevent retrograde regurgitation of the injected filler in the upward direction. Then, while standing in front of the patient, we injected the filler (approximately $0.1 \mathrm{ml}$ ) into the same zone through the wet-dry junction. After filler injection, both sides of the injected areas were gently pressed to make an oval shape. Subsequently, the same method was applied to the opposite side. Symmetry of both sides was checked, and filler was added to the asymmetric area.

The results in the three patients are shown in Fig. 3-5.

The mouth looks shortened transversely and more rounded due to the anterior projection of the centro-medial zone of the vermilion (Fig. 3). The whole contour of the upper vermilion border was significantly changed from flat to round and curved. The distance between the nasal base and upper lip appears shortened, which is caused by eversion and projection of the upper vermilion border (Fig. 3-5).

Although the position of the mouth corner was not changed, interestingly, the corner of mouth appears lifted, which is caused by the illusional effect of combining centro-medial volume restoration and changed lateral vermilion contour (Fig. 3-5). 

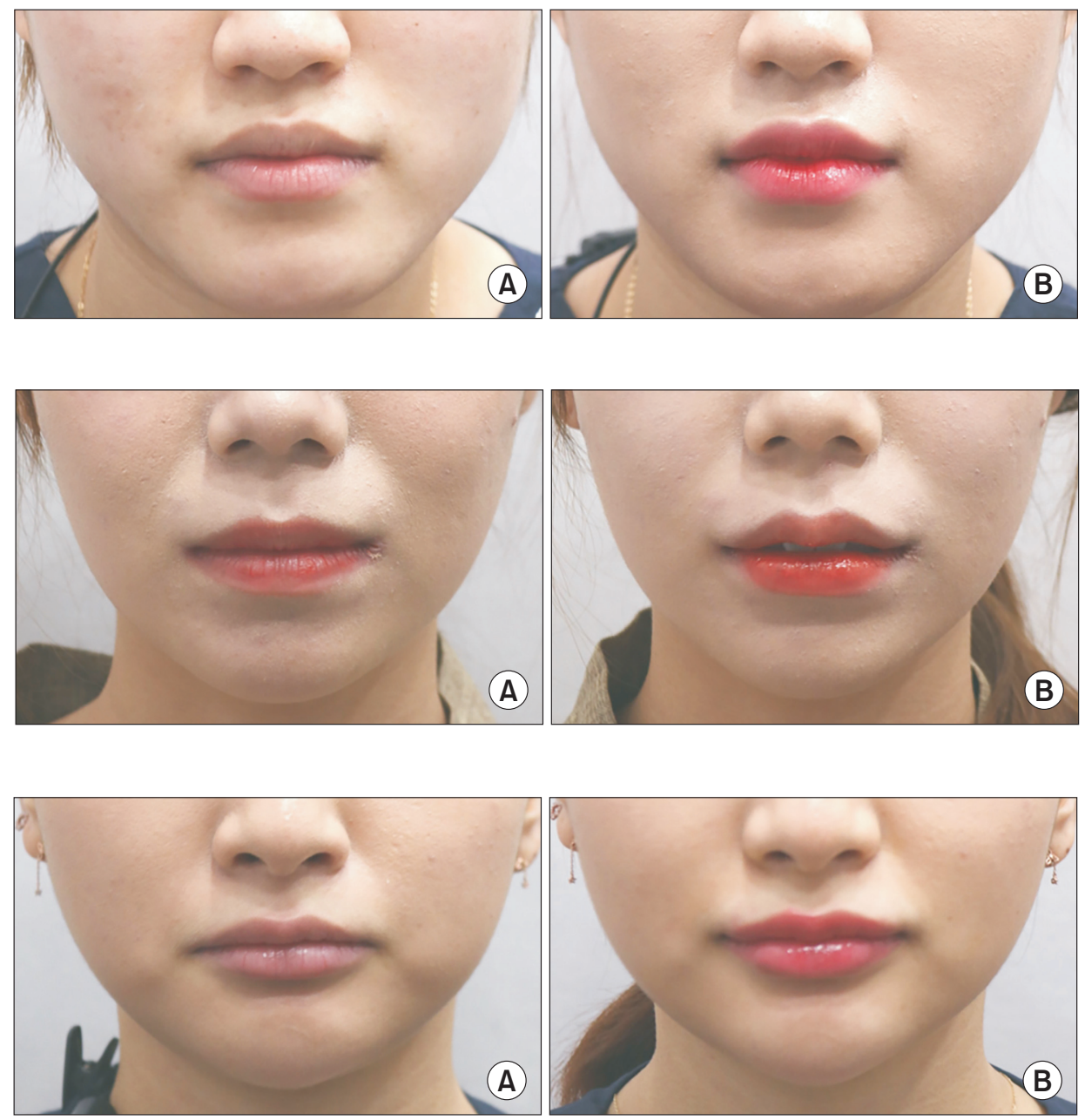

Fig. 3. Change in lip contour. Filler injection was performed in a 26-year-old woman who wanted to improve volume deficiency and ptotic lip appearance. Before (A) and 1-month after the procedure (B).

Fig. 4. Change in lip contour. Filler injection was performed in a 25-yearold woman who wanted to have lips with obvious contour and vibrant appearance. Before (A) and 1-month after the procedure (B).

Fig. 5. Change in lip contour. Filler injection was performed in a 21-year-old woman who wanted to improve volume deficiency and ptotic lip appearance while closing her mouth. Before (A) and 1-month after the procedure (B).
The flat upper vermilion contour was greatly changed to round and more obvious, similar to a bird-flying figure, after filler injection, leading to more natural and vibrant-looking lips with lifted appearance (Fig. 4).

A significant improvement in volume restoration with a natural and beautiful contour was observed. Particularly, oral and perioral contour while closing the mouth looks very natural and comfortable; moreover, the mouth corner looks lifted (Fig. 5).

An unnatural contour just above the upper lateral vermilion border was noted. It is believed to be caused by either retrograded regurgitation of the injected filler or hypercorrection. With the overall change in lip contour, the upper lips appeared lifted; however, regionally focusing on the lateral vermilion zone of the upper lip, it may look slightly ptotic (Fig. 4, 5).

\section{Discussion}

Lip contouring procedures, such as surgical correction, filler and botulinum toxin injection, and thread insertion, have been performed. Among them, surgical correction is not widely use because it results in scar and unnatural contour. Moreover, botulinum toxin injection has not shown sufficient and longlasting results. Traditional filler injection has some limitations in improving the contour of the lateral vermilion zone together with the mouth corner.

Traditional lip filler injection focuses on volume restoration and wrinkle removal. However, our procedure showed not only volumizing effect but also change in the whole lip contour with dynamic and vibrant appearance, as if the mouth corner is lifted [3]. The difference between the traditional method and our method was the additional filler injection for forming volumetric eversion to improve the rolling-in shape from the lateral vermilion zone, near the mouth corner, in the upper lip. It led to a change in the whole contour of the upper lip from flat and ptotic shape to oval and lifted shape. Particularly, the contour of the lateral vermilion zone in the upper lip showed smooth transition of convex-concave line near the mouth corner.

In young patients, like these three cases, the author's ap- 
proach may be suitable; however, in old patients who have severely drooped mouth corner, it is recommended that the procedure should be preferentially focused on volume restoration of the lip and philtral zone and removal of perioral wrinkles and folds as that in traditional lip filler injection.

Even though this procedure showed promising results, only with those of three cases, it is quite difficult to prove the real effect of this procedure by estimation of morphological changes. Therefore, it is necessary to perform the procedure with sufficient number of cases, and its result should be analyzed by measurement.

This procedure showed an entire change in the lip contour with volumizing effect and vibrant appearance and possibility of mouth corner lifting in three young women.

\section{Conflicts of interest}

The authors have nothing to disclose.

\section{References}

1. Sullivan PK, Hoy EA, Mehan V, Singer DP. An anatomical evaluation and surgical approach to the perioral mound in facial rejuvenation. Plast Reconstr Surg 2010;126:1333-40.

2. Rohrich RJ, Pessa JE. The anatomy and clinical implications of perioral submuscular fat. Plast Reconstr Surg 2009;124:26671.

3. Mannino GN, Lipner SR. Current concepts in lip augmentation. Cutis 2016;98:325-9. 